\title{
Teres Minor Muscle
}

National Cancer Institute

\section{Source}

National Cancer Institute. Teres Minor Muscle. NCI Thesaurus. Code C33751.

One of four rotator cuff muscles that form a musculotendinous cuff around the glenohumeral joint, which provides muscular support to the shoulder; it arises from the lateral border of the scapula with insertion on the greater tubercle of the humerus, and functions to laterally rotate the humerus. 